\title{
Histological Comparison of Post-extraction Alveolar Bone Repair Treated with Melatonin and Calcium Sulfate: An In Vivo Study in Cavia porcellus
}

\author{
Henrry Torres ${ }^{1}$, Franco Mauricio ${ }^{2}$, Roman Mendoza ${ }^{3}$, Daniel Alvitez-Temoche ${ }^{4}$, Julia Medina ${ }^{5}$, Frank Mayta-Tovalino ${ }^{6}$
}

\begin{abstract}
Aim and objective: To histologically compare alveolar bone repair after tooth extraction treated with melatonin and calcium sulfate in an in vivo experimental study in guinea pigs (Cavia porcellus).

Materials and methods: The study was of longitudinal, prospective, and experimental design in an animal bio-model. A total of 24 male guinea pigs were included, weighing from 700 to $900 \mathrm{~g}$ and separated into two experimental groups (melatonin and calcium sulfate) for three periods $(15,30$, and 45 days) at 15-day intervals after surgery. The guinea pigs were randomly included into groups for the time evaluated.

Results: In relation to bone repair cells using calcium sulfate, the presence of osteoblasts at 15,30 , and 45 days was $39.0 \pm 63,55.3 \pm 6.0$, respectively, with $61.3 \pm 10.0$ cells per field. Regarding bone repair cells using melatonin, the presence of osteoblasts at 15,30 , and 45 days was $25.0 \pm 3.7,49.3 \pm 1.5$, respectively, with $53.6 \pm 5.6$ cells per field.

Conclusion: Both melatonin and calcium sulfate were found to be useful in bone repair at a histological and clinical level, although they present certain nonsignificant, albeit marked advantages in the bone repair process when compared with the control socket at the histological level. Clinical significance: This research allows us to know the usefulness of these easily accessible chemicals for the generation of bone repair. Keywords: Bone repair, Calcium sulfate, Cavia porcellus, Melatonin.

The Journal of Contemporary Dental Practice (2021): 10.5005/jp-journals-10024-3150
\end{abstract}

\section{INTRODUCTION}

The insertion periodontium is a functional unit that is found around the tooth, the main role of which is to maintain the tooth firmly anchored in its physiological position. This unit constitutes a structural assembly made up of cement, periodontal ligament, and alveolar bone. ${ }^{1}$ When tooth loss due to avulsion (fortuitous or pathological) or therapeutic extraction occurs, the cement and part of the periodontal ligament, firmly anchored to the dentin surface, accompany the tooth, while the rest of the ligament and alveolar bone lose their anchoring function and are gradually reabsorbed. ${ }^{1-5}$

Knowledge of the histological structure of this bone tissue and its physiological mechanisms of structural maintenance is therefore essential to improve the rational use of new therapeutic technologies aimed at improving bone regeneration. 'Blood factors are involved in alveolar bone repair, including granulation tissue, of which fibroblasts are the most representative cells. The bone matrix is made up of osteoblasts, and mineralized bone includes the presence of osteoclasts. Bone repair processes involve bone production by osteoblasts and bone resorption by osteoclasts that consolidate over time. ${ }^{4-8}$

Osteoblasts are the cells that differentiate the synthesis of the organic matrix of bone which together make up the osteoid, after stimulation by parathormone, vitamin D, estrogens, and leptins. ${ }^{1}$ On the contrary, osteoclasts originate in the bone marrow and belong to the monocyte-macrophage system. The cytoplasm of osteoclasts measures 40-150 microns. It is acidophilic and has four to six nuclei and has a rough area on the contact surface with the bone trabecula, which is formed by numerous folds of the plasma membrane. ${ }^{2,10-12}$

\begin{abstract}
${ }^{1-3}$ Faculty of Dentistry, Department of Doctorate in Dentistry, Universidad Nacional Federico Villarreal, Lima, Peru

${ }^{4,5}$ Faculty of Dentistry, Academic Department, Universidad Nacional Federico Villarreal, Lima, Peru

${ }^{6}$ Faculty of Health Sciences, Postgraduate Department, CHANGE Research Working Group, Universidad Cientifica del Sur, Lima, Peru

Corresponding Author: Frank Mayta-Tovalino, Faculty of Health of Sciences, Postgraduate Department, CHANGE Research Working Group, Universidad Cientifica del Sur, Lima, Peru, Phone: +51 1 214-2500, e-mail: fmaytat@cientifica.edu.pe
\end{abstract}

How to cite this article: Torres H, Mauricio F, Mendoza R, et al. Histological Comparison of Post-extraction Alveolar Bone Repair Treated with Melatonin and Calcium Sulfate: An In Vivo Study in Cavia porcellus. J Contemp Dent Pract 2021;22(7):739-744.

Source of support: Nil

Conflict of interest: None

The literature has mentioned that melatonin could have effects on bone healing since it has a protective effect due to its ability to regulate the antioxidant effects of osteogenic cells, in addition to promoting angiogenesis. ${ }^{8-10}$ The bone matrix is made up of two main components: an organic matrix that represents $35 \%$ of the dry weight and an inorganic component that represents $65 \%$. The organic portion of the matrix, or osteoid, is made up of collagen fibers, mainly type I (90\%), in the form of large bundles, and it also contains small proportions of type III and IV collagen. The remaining $10 \%$ is made up of noncollagen substances, $8 \%$ of which are glycoproteins, phosphoproteins, and proteoglycans. The remaining $2 \%$ is represented by enzymes (alkaline phosphatase, 
collagenase, among others), extravasated products from the blood, and by growth factors (the osteoinductive factor-osteogenin-, transforming growth factor beta, fibroblast growth factor, among others) that have part of their reservoir in the bone matrix. ${ }^{3-5}$ After extraction, the socket undergoes a series of processes to preserve the alveolar dimension and avoid reabsorption which could be detrimental for subsequent repair with implants or for making removable prostheses.

The purpose of this study is to histologically compare alveolar bone repair after tooth extraction treated with melatonin and calcium sulfate in an in vivo experimental study in guinea pigs (Cavia porcellus).

\section{Materials and Methods Design and Sample Size}

The study was of longitudinal, prospective, and experimental design in an animal bio-model (C. porcellus). A pilot test was carried out for each period and according to the results obtained. The sample size was calculated using the two-means comparison formula with Software Stata ${ }^{\otimes} 15$ (Texas, USA), for which an alpha of 0.05 and a beta of 0.8 were considered.

\section{Allocation}

The animals were caged and coded by a single operator. The entire study was carried out by double-blindness. A total of 24 male guinea pigs (C. porcellus) weighing within the range of 700-900 g. The animals were randomly separated by simple random sampling into two experimental groups (melatonin and calcium sulfate) for three periods (15, 30, and 45 days) at 15-day intervals after surgery and were transferred to the operating room of the Faculty of Zootechnics of the Universidad Nacional Agraria La Molina. The following groups were formed (Flowchart 1):

First period (15 days): Eight C. porcellus Group I: C. porcellus receiving calcium sulfate. Group II: C. porcellus receiving melatonin.

Second period (30 days): Eight C. porcellus Group III: $C$. porcellus receiving calcium sulfate. Group IV: C. porcellus receiving melatonin.
Third period (45 days): Eight C. porcellus

Group V: C. porcellus receiving calcium sulfate.

Group VI: C. porcellus receiving melatonin.

Inclusion Criteria

- C. porcellus with good general condition.

- 1 year of age.

- Weight ranging between $0.7 \mathrm{~kg}$ and $1.2 \mathrm{~kg}$.

- Male sex.

\section{Exclusion Criteria}

- C. porcellus with a poor general condition or with some physical alteration.

- C. porcellus that do not conform to ad libitum feeding.

\section{Preparation of Calcium Sulfate for Grafting}

About $250 \mathrm{~g}$ of calcium sulfate powder was collected in a steel container and sterilized by dry heat in a hot air oven at $121^{\circ} \mathrm{C}$ for 15 minutes and then stored in a sterile package. Half an hour before surgery, the calcium sulfate was mixed with absolute alcohol to make a pasty material, ensuring additional sterilization of the powder. Following evaporation of the alcohol after 30 to 45 minutes, the calcium sulfate became a dry powder. Once the dry powder was ready, $1 \mathrm{~g}$ of ceftriaxone powder was added and mixed followed by distilled water to make a thick paste powder to be incorporated into the postextraction cavity.

\section{Preparation of Melatonin for Grafting}

One gram tablets of pure melatonin without excipients were crushed to the consistency of a powder and then diluted with distilled water to make a pasty consistency. Like the preparation of calcium sulfate, $70 \%$ alcohol and $1 \mathrm{~g}$ of ceftriaxone were added to the mixture to produce a cement powder.

\section{Tooth Extraction}

All the animals $(n=24)$ underwent extraction of the mandibular premolars from both sides. According to the study group, the experimental material was placed in the right socket of each animal and the left socket served as control. For molar extraction, each animal was anesthetized with an intraperitoneal dose of $30 \mathrm{mg} / \mathrm{kg}$ of sodium pentobarbital. Each C. porcellus was then wrapped in a

Flowchart 1: Flow diagram shows study protocol

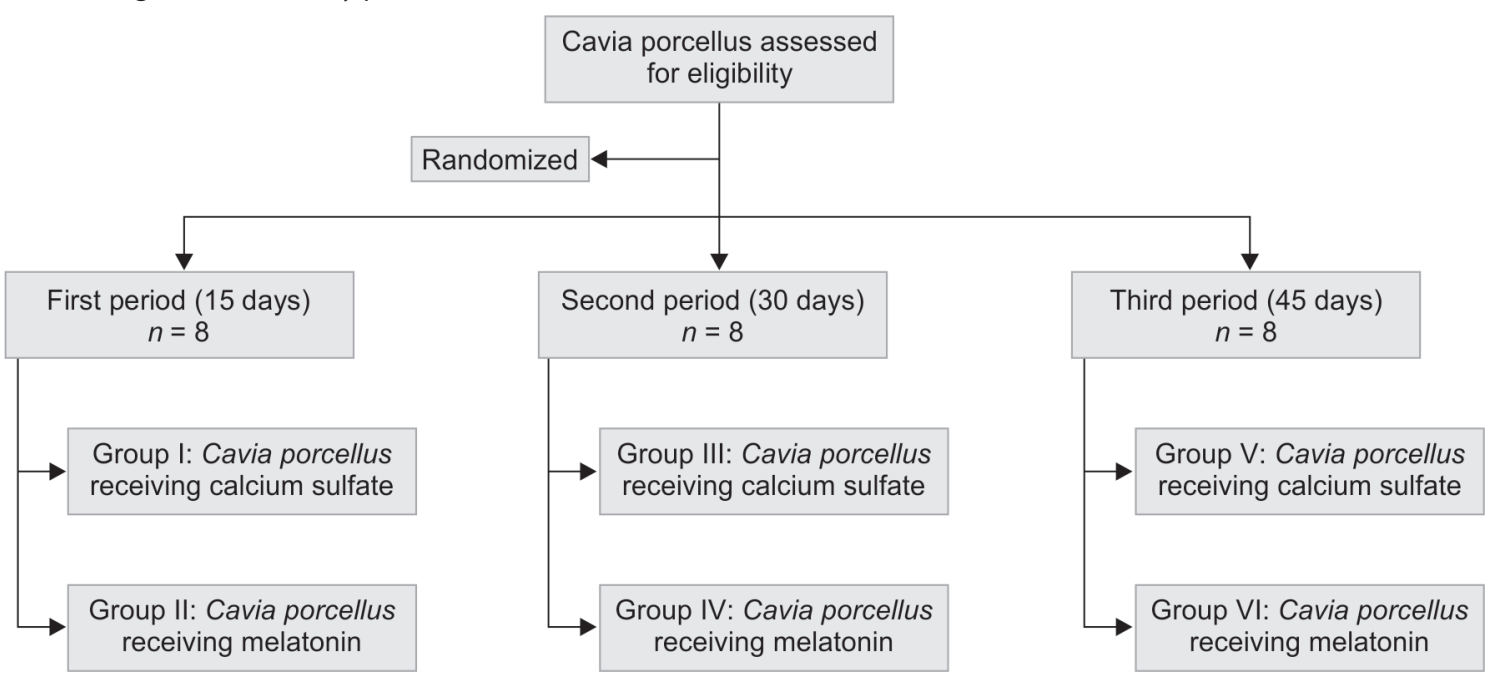


surgical field and the mouth was kept open with adapted surgical separators. For the extraction of the teeth, an intrasulcular incision was made around the first right and left mandibular molars using a number 15 scalpel blade, and a full-thickness flap was then lifted. Exodontia was performed using an elevator for deciduous teeth conditioned with a $3 \mathrm{~mm}$ active part and with Dove tooth forceps for deciduous teeth. The melatonin or calcium sulfate material was randomly placed inside one of the alveoli and the wound was sutured with Vicryl (RB1-5/0). The contralateral dental socket that served as the control was washed with distilled water. The animals were sacrificed by anesthetic overdose at 15, 30, and 45 days postextraction (Fig. 1).

\section{Histological Procedure}

After euthanasia, the jaw of the animal was removed and both hemiarcates were separated. The following procedures were then performed: (a) Fixation: The samples obtained from the dental alveoli of the C. porcellus jaw were placed in glass jars with $10 \%$ formalin. They were then subjected to a decalcification process with $5 \%$ nitric acid for a period of 20 days. (b) Dehydration: The fixed tissue was placed

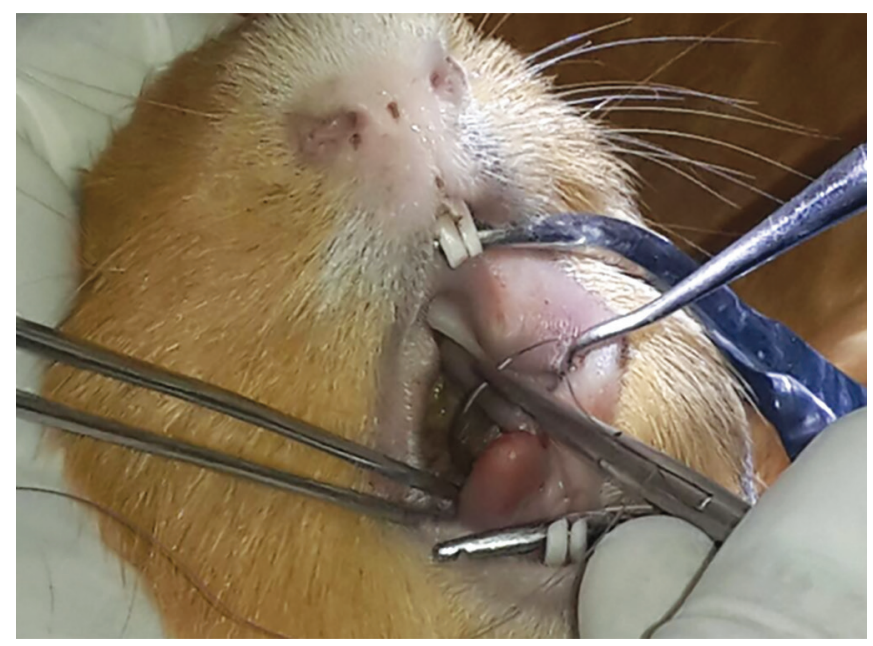

Fig. 1: Tooth extraction

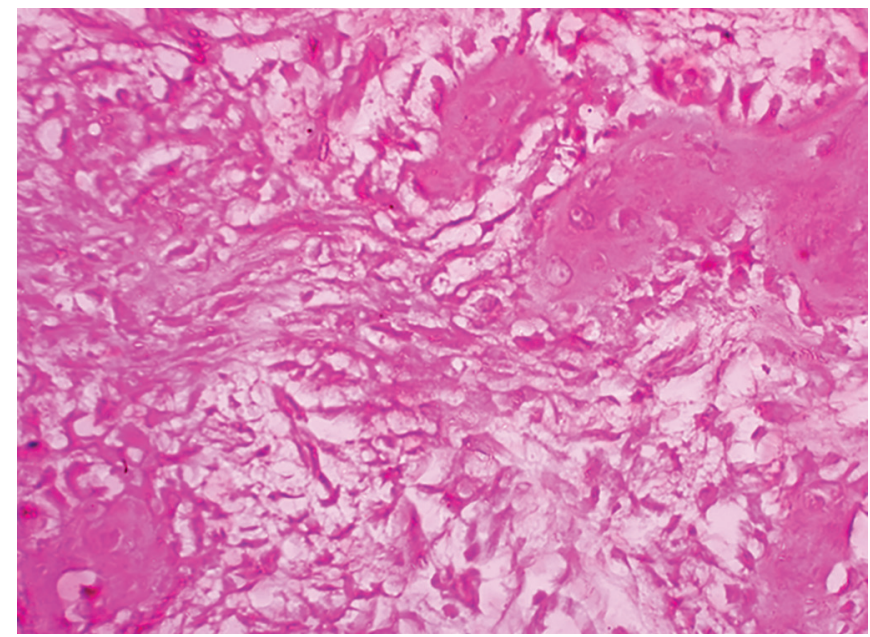

Fig. 2: (Group I) Optical photomicrograph of alveolar bone with hematoxylin-eosin staining. $400 \times$ magnification. Osteoblasts and bone matrix, osteocytes, and fibroblasts are observed in alcohol of increasing degrees starting with $50 \%$ until reaching absolute alcohol. Subsequently, the clearance process was carried out consisting of placing the piece in Xylol (an intermediate between ethanol and paraffin). The piece was embedded in paraffin until a block was obtained. (c) In object holders, the paraffin block was cut into slices between 5 and $10 \mu \mathrm{m}$ thick, which were placed in a water bath. The slices on the slides were then stained with hematoxylin and eosin. (d) Histological analysis was performed by blind reading using a multihead light microscope (Olympus, model BX50F4, Olympus Optical Co, Ltd. Japan) at 400× magnification. Two fields were chosen: one in the apical area of the socket and the other in the middle area, recorded by microphotographs (Figs 2 and 3).

\section{Ethical Considerations}

This study was reviewed by the graduate office of the School of the National University Federico Villarreal with approval code 192-2017-OG-EUPG-UNFV. Additionally, the writing of this manuscript was prepared following The ARRIVE guidelines (Animal Research: Reporting of In Vivo Experiments) as a checklist of recommendations to improve the reporting of research involving animals.

\section{Statistical Analysis}

Summary measures tables (mean, median, 25th percentile, 75th percentile, and standard deviation) were prepared to determine the distribution of the data for each measure (fibroblasts, osteoblasts, and trabeculae) at each time. To compare between groups, the Wilcoxon rank-sum test was used for each of the measurements and times, with a significance level of 0.05 . The database was made in Excel v2010. Data processing and analysis were performed with the Stata 15 statistical program (Texas, USA).

\section{Results}

In relation to the apical third, the presence of osteoblasts at 15 , 30 , and 45 days was $39.0 \pm 63,55.3 \pm 6.0$, and $61.3 \pm 10.0$ cells per field, respectively. However, the measurements in relation to the middle third were relatively lower with $36.2 \pm 16.8,44.6 \pm 4.9$, and $52.0 \pm 14.4$ cells per field at 15,30 , and 45 days, respectively. The inferential analysis only showed statistically significant differences

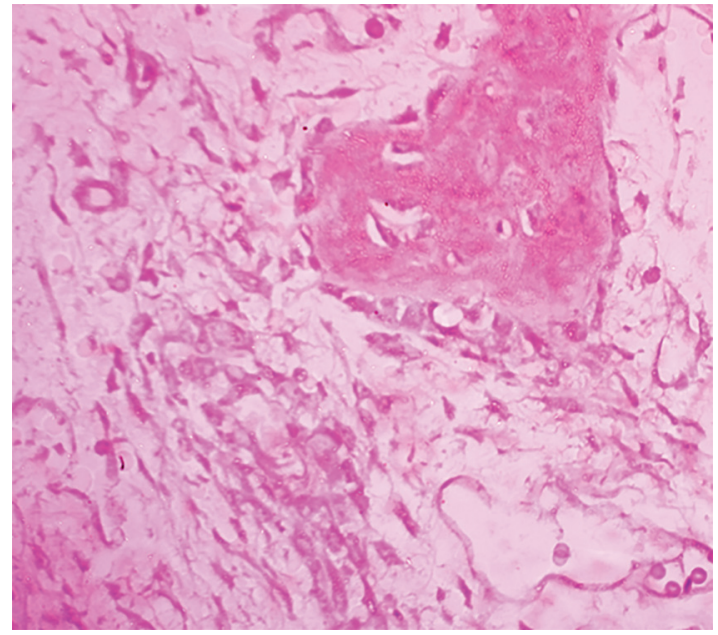

Fig. 3: (Group II) Optical photomicrograph of alveolar bone with hematoxylin-eosin staining. $400 \times$ magnification. Osteoblasts and bone matrix, osteocytes, and fibroblasts are observed 
at 30 and 45 days in the middle and apical thirds, respectively, with a $p<0.05$ (Table 1).

In relation to the apical third, the presence of osteoblasts at 15 , 30 , and 45 days was $25.0 \pm 3.7,49.3 \pm 1.5$, and $53.6 \pm 5.6$ cells per field, respectively, while the measurements related to the middle third were relatively heterogeneous at 15,30 , and 45 days with $17.3 \pm 4.5,51.3 \pm 2.5$, and $49.0 \pm 4.0$ cells per field, respectively. The inferential analysis only showed statistically significant differences at 15 and 30 days in the apical and middle thirds, respectively, with a $p<0.05$ (Table 2).

Table 1: Bone repair cells according to time and alveolar third using calcium sulfate

\begin{tabular}{|c|c|c|c|c|c|}
\hline Time & Section & Cell & Mean & $S D$ & $p$ \\
\hline \multirow[t]{6}{*}{ Day 15} & Apical third & Fibroblasts & 31.7 & 4.5 & $>0.05$ \\
\hline & & Osteoblasts & 39.0 & 6.3 & \\
\hline & & Trabeculae & 2.0 & 0.0 & \\
\hline & Middle third & Fibroblasts & 39.0 & 2.9 & $>0.05$ \\
\hline & & Osteoblasts & 36.2 & 16.8 & \\
\hline & & Trabeculae & 1.7 & 0.9 & \\
\hline \multirow[t]{6}{*}{ Day 30} & Apical third & Fibroblasts & 49.6 & 8.3 & $>0.05$ \\
\hline & & Osteoblasts & 55.3 & 6.0 & \\
\hline & & Trabeculae & 2.3 & 1.1 & \\
\hline & Middle third & Fibroblasts & 51.0 & 2.0 & 0.036 \\
\hline & & Osteoblasts & 44.6 & 4.9 & \\
\hline & & Trabeculae & 1.6 & 0.5 & \\
\hline \multirow[t]{6}{*}{ Day 45} & Apical third & Fibroblasts & 30.6 & 1.5 & 0.033 \\
\hline & & Osteoblasts & 61.3 & 10.0 & \\
\hline & & Trabeculae & 2.3 & 0.5 & \\
\hline & Middle third & Fibroblasts & 38.3 & 17.6 & $>0.05$ \\
\hline & & Osteoblasts & 52.0 & 14.4 & \\
\hline & & Trabeculae & 2.6 & 0.5 & \\
\hline
\end{tabular}

Table 2: Bone repair cells according to time and alveolar third using melatonin

\begin{tabular}{|c|c|c|c|c|c|}
\hline Time & Section & Cell & Mean & $S D$ & $p$ \\
\hline \multirow[t]{6}{*}{ Day 15} & Apical third & Fibroblasts & 33.2 & 1.7 & 0.04 \\
\hline & & Osteoblasts & 25.0 & 3.7 & \\
\hline & & Trabeculae & 1.2 & 0.5 & \\
\hline & Middle third & Fibroblasts & 26 & 4.0 & $>0.05$ \\
\hline & & Osteoblasts & 17.3 & 4.5 & \\
\hline & & Trabeculae & 1.6 & 0.4 & \\
\hline \multirow[t]{6}{*}{ Day 30} & Apical third & Fibroblasts & 34.3 & 5.6 & 0.036 \\
\hline & & Osteoblasts & 49.3 & 1.5 & \\
\hline & & Trabeculae & 2.0 & 1.0 & \\
\hline & Middle third & Fibroblasts & 39.3 & 6.6 & 0.036 \\
\hline & & Osteoblasts & 51.3 & 2.5 & \\
\hline & & Trabeculae & 2.0 & 0.0 & \\
\hline \multirow[t]{6}{*}{ Day 45} & Apical third & Fibroblasts & 33.3 & 4.0 & $>0.05$ \\
\hline & & Osteoblasts & 53.6 & 5.6 & \\
\hline & & Trabeculae & 2.0 & 0.0 & \\
\hline & Middle third & Fibroblasts & 42.3 & 4.1 & $>0.05$ \\
\hline & & Osteoblasts & 49 & 4.0 & \\
\hline & & Trabeculae & 2.0 & 0.0 & \\
\hline
\end{tabular}

In relation to the apical third, the presence of osteoblasts at 15,30 , and 45 days was $24.7 \pm 9.2,42.0 \pm 0.0$, and $45.5 \pm 14.3$ cells per field, respectively. However, the measurements of the middle third were relatively lower at 15,30 , and 45 days with $22.5 \pm 3.8$, $44.0 \pm 0.0$, and $44.7 \pm 5.3$ cells per field, respectively (Table 3 ).

\section{Discussion}

The present study histologically compared the effects of melatonin and calcium sulfate on the alveolar bone repair process after tooth extraction. The results demonstrate that the alveolar bone repair process involves a series of events that include the formation of a provisional matrix and a mineralized bone. The indicators used in the present study to assess alveolar bone repair were similar to those described elsewhere. ${ }^{13-15}$

The results show a series of similarities compared to other studies including bone formation with and without the application of the experimental material, and the samples were very homogeneous and compact with the calcium sulfate compared to the control socket. The coagulated alveoli showed statistically less bone formation than the defect presented by the calcium sulfate filling. The mean bone formation of the trabecular bone area was greater in the grafts than in the control group. Greater provisional matrix was observed at 14 days in the sockets with calcium sulfate compared to the control sockets and this was maintained at 30 days.

According to the formation of granulation tissues, bone matrix, and mineralized bone in the socket receiving melatonin compared to the control socket, the number of fibroblasts increased up to 30 days, in both the apical and middle areas. The values at 45 days were like those at 15 days. In the bone matrix, osteoblasts were found to increase on both sides at 45 days. There were significant differences compared to the control socket in relation to tissue granulation with the presence of fibroblasts at 15 and 30 days in the apical and middle areas. Regarding the bone matrix, there was a significant difference in the medial and apical area at 30 days, with

Table 3: Bone repair cells according to time and alveolar third in the control socket

\begin{tabular}{|c|c|c|c|c|}
\hline Time & Section & Cell & Mean & $S D$ \\
\hline \multirow[t]{6}{*}{ Day 15} & Apical third & Fibroblasts & 32.5 & 7.1 \\
\hline & & Osteoblasts & 24.7 & 9.2 \\
\hline & & Trabeculae & 2.0 & 0 \\
\hline & Middle third & Fibroblasts & 29.0 & 11.8 \\
\hline & & Osteoblasts & 22.5 & 3.8 \\
\hline & & Trabeculae & 1.2 & 0.5 \\
\hline \multirow[t]{6}{*}{ Day 30} & Apical third & Fibroblasts & 45.0 & 0 \\
\hline & & Osteoblasts & 42.0 & 0 \\
\hline & & Trabeculae & 2.0 & 0 \\
\hline & Middle third & Fibroblasts & 46.0 & 0 \\
\hline & & Osteoblasts & 44.0 & 0 \\
\hline & & Trabeculae & 2.0 & 0 \\
\hline \multirow[t]{6}{*}{ Day 45} & Apical third & Fibroblasts & 42.7 & 8.8 \\
\hline & & Osteoblasts & 45.5 & 14.3 \\
\hline & & Trabeculae & 2.0 & 0.8 \\
\hline & Middle third & Fibroblasts & 34.7 & 18.2 \\
\hline & & Osteoblasts & 44.7 & 5.3 \\
\hline & & Trabeculae & 2.2 & 0.5 \\
\hline
\end{tabular}


a significant presence of trabeculae in the apical area at 15 days. These results are similar to a previous study 14-17 which observed that the presence of bone matrix is an important mediator in bone formation and stimulation and that melatonin generates a greater presence of fibroblasts and osteoblasts and a lower presence of osteoclasts, promoting bone integration. It should be noted that in the immediate postoperative period there is a significant increase in oxidative stress that is counteracted by melatonin. ${ }^{14-17}$

In relation to the utility of a coating material for bone repair following a tooth extraction, there are certain advantages in terms of the infiltration of bone repair cells compared to the coagulum of an alveolus. On comparing which components have the most beneficial effect on alveolar bone consolidation, no significant differences were observed over time. However, each material showed certain activities related to bone repair, favoring bone remodeling and permanence. Melatonin is an important component related to oxidation, retaining cellular fibroblasts for bone maintenance, and calcium sulfate strengthens and consolidates the trabecular framework in the stages of bone repair after 30 days. $8,14,15$

The extrapolation of results obtained in experimental animals to the human species is debatable, especially taking into account that different studies have demonstrated that the bone regeneration process is faster in these animals. ${ }^{16-19}$ The present study showed the increasing presence of fibroblasts as an indicator of granulation tissue in the bone repair process at days 15 and 30, although the number of these cells decreased at 45 days, similar to the results of previous studies. ${ }^{12-15,20}$ A longer evaluation period could provide more information for evaluating the alveolar repair process since this phenomenon of change takes place over a longer period of time.

The present study is relevant due to the scarce literature evaluating the differential behavior of melatonin compared to calcium sulfate in the alveolar bone. In the absence of studies in this regard, greater knowledge of the direct actions of melatonin on alveolar bone in relation to immunomodulators, nitric oxide decreasing capacity, angiogenic capacity, repair capacity, anti-inflammatory capacity, protection against infections, and antinociceptive capacity is needed. In addition, it is necessary to investigate the local effects and the histopathological changes of melatonin and calcium sulfate as possible new therapeutic agents in conservative dentistry when used as a coating directly applied on the alveolus. Lastly, it is of great interest to demonstrate the effectiveness of calcium sulfate and/or melatonin on bone regeneration following tooth extraction.

However, this research was partially limited by a socioeconomic type of factor, being an experimental work that involves multidisciplinary work and professional external advice and the little recognition and investment of private and state companies in the development of new knowledge for this type of study. Another limitation is technical and specialized management in the histopathological field, which will greatly depend on the suggestion and advice of other professionals. Similarly, the management and conservation of experimental animals would be a limitation for the researcher, who will depend in part on the veterinary professional who will oversee providing the knowledge and requirements necessary for their conservation and management. Finally, the biggest limitations of the present study include the need for specialized technical histopathological knowledge and advice as well as veterinary professionals to ensure adequate care of the study animals.

\section{Conclusion}

The use of calcium sulfate for alveolar bone repair post-tooth extraction increases fibroblast production in both the apical and middle thirds for up to 30 days, while at 45 days these values are similar to those at 15 days. An increase in osteoblasts was observed at 30 and 45 days on both sides of the mouth; however, no changes in trabeculae were observed. Both melatonin and calcium sulfate were found to be useful in bone repair at both a histological and clinical level, although at a histological level, they present certain nonsignificant, albeit marked, advantages in the bone repair process when compared with the control socket.

\section{References}

1. Boyle WJ, Simonet WS, Lacey DL. Osteoclast differentiation and activation. Nature 2003;423(6937):337-342. DOI: 10.1038/ nature01658.

2. Hadjidakis DJ, Androulakis II. Bone remodeling. Ann N Y Acad Sci 2006;1092:385-396. DOI: 10.1196/annals.1365.035.

3. Huiskes R. If bone is the answer, then what is the question? J Anat 2000;197(Pt 2):145-156. DOI: 10.1046/j.1469-7580.2000. 19720145.x.

4. Mano T, Akita K, Fukuda N, et al. Histological comparison of three apatitic bone substitutes with different carbonate contents in alveolar bone defects in a beagle mandible with simultaneous implant installation. J Biomed Mater Res B Appl Biomater 2020;108(4): 1450-1459. DOI: 10.1002/jbm.b.34492.

5. Sargolzaie N, Rafiee M, Salari Sedigh H, et al. Comparison of the effect of hemihydrate calcium sulfate granules and Cerabone on dental socket preservation: an animal experiment. J Dent Res Dent Clin Dent Prospects 2018;12(4):238-244. DOI: 10.15171/ joddd.2018.037.

6. Hao F, Qin L, Liu J, et al. Assessment of calcium sulfate hemihydratetricalcium silicate composite for bone healing in a rabbit femoral condyle model. Mater Sci Eng C Mater Biol Appl 2018;88:53-60. DOI: 10.1016/j.msec.2018.02.024.

7. Artas G, Gul M, Acikan I, et al. A comparison of different bone graft materials in peri-implant guided bone regeneration. Braz Oral Res 2018;32:e59. DOI: 10.1590/1807-3107bor-2018.vol32.0059.

8. Cutando A, Gómez-Moreno G, Arana C, et al. Melatonin stimulates osteointegration of dental implants. J Pineal Res 2008;45(2):174-179. DOI: 10.1111/j.1600-079X.2008.00573.x.

9. Guardia J, Gómez-Moreno G, Ferrera MJ, et al. Evaluation of effects of topic melatonin on implant surface at 5 and 8 weeks in Beagle dogs. Clin Implant Dent Relat Res 2011;13(4):262-268. DOI: 10.1111/j.17088208.2009.00211.x.

10. Calvo-Guirado JL, Gómez-Moreno G, López-Marí L, et al. Actions of melatonin mixed with collagenized porcine bone versus porcine bone only on osteointegration of dental implants. J Pineal Res 2010;48(3):194-203. DOI: 10.1111/j.1600-079X.2009.00743.x.

11. Calvo-Guirado JL, Gómez-Moreno G, Barone A, et al. Melatonin plus porcine bone on discrete calcium deposit implant surface stimulates osteointegration in dental implants. J Pineal Res 2009;47(2):164-172. DOI: 10.1111/j.1600-079X.2009.00696.x.

12. Hu MH, Lee PY, Chen WC, et al. Comparison of three calcium phosphate bone graft substitutes from biomechanical, histological, and crystallographic perspectives using a rat posterolateral lumbar fusion model. Mater Si Eng C Mater Biol Appl 2014;45:82-88. DOI: 10.1016/j.msec.2014.08.065.

13. Kutkut A, Andreana S, Kim HL, Monaco E Jr. Extraction socket preservation graft before implant placement with calcium sulfate hemihydrate and platelet-rich plasma: a clinical and histomorphometric study in humans. J Periodontol. 2012 Apr;83(4):401-9. DOI: 10.1902/ jop.2011.110237. 
14. Cutando A, Arana C, Gómez-Moreno G, et al. Local application of melatonin into alveolar sockets of beagle dogs reduces tooth removal-induced oxidative stress. J Periodontol 2007;78(3):576-583. DOI: 10.1902/jop.2007.060244.

15. Schlickewei CW, Laaff G, Andresen A, et al. Bone augmentation using a new injectable bone graft substitute by combining calcium phosphate and bisphosphonate as composite-an animal model. J Orthop Surg Res 2015;10:116. DOI: 10.1186/s13018-015-0263-z.

16. Li JJ, Dunstan CR, Entezari A, Li Q, et al. A novel bone substitute with high bioactivity, strength, and porosity for repairing large and loadbearing bone defects. Adv Healthc Mater 2019;8(8):e1801298. DOI: 10.1002/adhm.201801298.

17. Sugawara A, Fujikawa K, Takagi S, Chow LC. Histological analysis of calcium phosphate bone grafts for surgically created periodontal bone defects in dogs. Dent Mater J. 2008 Nov;27(6):787-94. DOI: 10.4012/dmj.27.787.

18. Busenlechner D, Tangl S, Mair B, et al. Simultaneous in vivo comparison of bone substitutes in a guided bone regeneration model. Biomaterials 2008;29(22):3195-3200. DOI: 10.1016/j. biomaterials.2008.04.021.

19. Anbu RT, Suresh V, Gounder R, et al. Comparison of the efficacy of three different bone regeneration materials: an animal study. Eur J Dent 2019;13(1):22-28. DOI: 10.1055/s-0039-1688735.

20. Macedo RM, Lacerda SA, Okamoto R, et al. Vital bone formation after grafting of autogenous bone and biphasic calcium phosphate bioceramic in extraction sockets of rats: histological, histometric, and immunohistochemical evaluation. Implant Dent 2018;27(6):615-622. DOI: 10.1097/ID.0000000000000815. 